\title{
High Frequency Oscillatory Ventilation Versus Conventional Ventilation in Management of Neonatal Meconium Aspiration syndrome
}

\author{
A.M.Shaheen , R.A.El-Sayed and A.S.Ali \\ Pediatrics and Neonatology Dept., Faculty of Medicine, Benha Univ., Benha, Egypt \\ E-Mail:Ahmed155@gmail.com
}

\begin{abstract}
Meconium aspiration syndrome (MAS) is complex respiratory disease of the term and near-term neonate, which keeps on putting a significant weight on neonatal escalated care assets around the world. The point of this examination was appraisal of high recurrence oscillatory ventilation versus traditional ventilation in administration of neonatal meconium desire, Method: This imminent randomized controlled preliminary included 50 infants admitted to NICU with meconium yearning (MAS), were isolated into 2 gatherings: Group 1: 25 cases treated by customary mechanical ventilation (CMV), Group 2: 25 cases treated by high-recurrence oscillatory ventilator (HFOV). All included patients were exposed to full history taking, total clinical assessment, and research center appraisal. Changes in the oxygenation work markers was utilized for checking patients' reaction as: Fraction of motivation $\mathrm{O} 2$ (FiO2), Oxygenation list (OI), blood vessel oxygen pressure/alveolar blood vessel oxygen strain (a/ApO2), at 2, 12, 24, $48 \mathrm{~h}$ of treatment. Results: This investigation included 27 guys (54\%) and 23 females (46\%), the mean gestational age was 37.82 \pm 2.6 weeks, CMV gathering, had a measurably longer span of ventilation time (CMV: 7.24 \pm 0.65 days, HFOV: $4.23 \pm 1.37$ days, $\mathrm{p}<0.001$ ), longer oxygen treatment (CMV: 15.39 \pm 2.44 days, HFOV: $11.76 \pm 5.32$ days, $\mathrm{p}<0.001)$, and longer season of hospitalization (CMV: $22.17 \pm 4.53$ days, HFOV: 17.92 \pm 12.6 days, $\mathrm{p}<0.001)$. End: Our outcomes demonstrated that HFOV seems to offer extra favorable circumstances contrasted with CMV in the treatment of MAS, by lessening the length of mechanical ventilation, and span of hospitalization, anyway it didn't influence the death rate.
\end{abstract}

Keywords: HFOV, CMV, MAS, Meconium, Aspiration.

\section{Introduction}

Meconium desire condition (MAS) is perhaps the most widely recognized reasons for extreme respiratory disappointment in newborn children conceived at term or post-term incubation, and furthermore one of the genuine sicknesses during the neonatal period where the embryo breathes in meconium-stained amniotic liquid (MSAF), preceding conveyance or during the conveyance, causing mechanical deterrent and synthetic aggravation along the respiratory lot and the alveolar, thus all the while causing other organ harms [1], [2].

Meconium staining of the amniotic liquid happens in around $10 \%$ to $20 \%$ of all term conveyances, however MAS happens in less than $33 \%$ of these babies [3]. Be that as it may, RY and Rao A have announced, about $10 \%$ MAS brought about respiratory disappointment in agricultural nations, the death rate was up to $39 \%$ [4].

Lung brokenness in MAS is a variable interchange of a few pathophysiological unsettling influences, boss among which are aviation route impediment, atelectasis, and aspiratory hypertension. Meconium, the viscid pigmented discharge of the fetal intestinal lot, is a harmful substance when breathed in, delivering one of the most exceedingly terrible types of goal pneumonitis experienced in people [5].

The most unmistakable and steady physiological impacts coming about because of meconium injury are hypoxaemia and diminished lung consistence. Some level of hypoxaemia is all inclusive in suggestive MAS, added to by numerous individuals of the previously mentioned poisonous impacts of meconium. Unsettling influences of oxygenation in MAS may identify with atelectasis, overdistension, aspiratory hypertension, or a mix of these. A difficult part of the administration of MAS is to perceive which system of hypoxaemia is the dominating one in some random newborn child at some random time. Especially where there is unmistakable aviation route impediment or articulated atelectasis, hypoxaemia might be joined by respiratory acidosis with $\mathrm{CO} 2$ maintenance identified with hypoventilation [6].

Hence, around $33 \%$ of infants with MAS require mechanical ventilation treatment [1]. In spite of over forty years of mechanical ventilation for babies with MAS, the ventilatory administration of the condition remains generally in the domain of "craftsmanship" instead of "science", with not many clinical preliminaries whereupon to base conclusive suggestions. Physiological standards and distributed experience do, notwithstanding, permit some core values to be advanced for regular ventilation system in MAS [6].

HFOV is viewed as a defensive system for human lungs, and utilizes a more modest or around a similar flowing volume as the anatomical dead space and ventilates at a higher recurrence (more or roughly multiple times the ordinary recurrence), accomplishing an uncommon new sort of ventilation with more powerful gas trade [7].

Regardless of the shortage of clinical preliminary, proof recommending an advantage, high recurrence oscillatory ventilation (HFOV) has become a significant methods for offering respiratory help for babies with extreme MAS bombing regular ventilation. Distributed arrangement from enormous neonatal information bases propose that $20-30 \%$ of all newborn children requiring intubation and ventilation with MAS are treated with 
high-recurrence ventilation, with the vast majority of these getting HFOV instead of high-recurrence stream ventilation (HFJV) [8].

Signs for changing to HFOV incorporate progressing hypoxaemia or potentially high $\mathrm{FiO} 2$, and, less generally, respiratory acidosis. In newborn children with critical atelectasis, satisfactory lung enlistment may require the utilization of a mean aviation route pressure $(\mathrm{P} A W)$ significantly higher than that on regular ventilation (up to $25 \mathrm{~cm} \mathrm{H} 2 \mathrm{O}$ now and again), with a stepwise enrollment move prone to be the best. Whenever oxygenation has improved, P AW should then be diminished; most babies with MAS requiring HFOV can be settled utilizing a $\mathrm{P}$ AW around 16$20 \mathrm{~cm} \mathrm{H} 2 \mathrm{O}$, with slow weaning in the days from that point [9].

Babies with noticeable gas catching may endure the enlistment cycle inadequately, with decreases in oxygenation and foundational circulatory strain and the potential for fuel of aspiratory hypertension. Enrollment moves of some structure can in any case be favorable in this gathering, with the advantage turning out to be evident when the P AW is diminished [10].

The point of this examination was evaluation of high recurrence oscillatory ventilation versus ordinary ventilation in administration of neonatal meconium aspiration.

\section{Patients and methods}

This prospective randomized controlled trial, included fifty newborns admitted to NICU of Benha university hospitals and Benha Children Hospital with meconium aspiration (MAS).

\section{Inclusion criteria}

- Both sexes (Males and females).

- The diagnosis of MAS was defined according to the following criteria, which basically consisted of history of fetal distress or asphyxia during delivery; thick meconium-stained amniotic fluid; meconium particles visible below the glottis; breathing difficulties combined with type II respiratory failure soon after birth; thoracic X-ray with pulmonary granular and patchy shadows [11].

\section{Exclusion criteria}

- The presence of major congenital malformations: (ie, congenital heart, cerebral, lung, abdominal malformations).

- Fetal hydrops

- Lack of parental consent

\section{Grouping}

According to the different approach of treatment, the patients were divided into 2 groups:

- Group 1: 25 cases treated by conventional mechanical ventilation (CMV)

- Group 2: 25 cases treated by high-frequency oscillatory ventilator (HFOV)

A standardized data sheet was utilized to record perinatal history, clinical examination and investigations that performed,

\section{Outcome measures, included:}

- Duration of assisted ventilation

- Duration of hospitalization

- Need of surfactant

- Occurrence bronchopulmonary dysplasia (BPD)

- Mortality rate

\subsection{Statistical analysis}

The data were coded, entered and processed on computer using SPSS (version 24). The results were represented in tabular and diagrammatic forms then interpreted. Mean, standard deviation, range, frequency, and percentage were use as descriptive statistics. The following tests were done: Chi-Square test $\mathrm{X}^{2}$ was used to test the association variables for categorical data. Student's t-test was used to assess the statistical significance of the difference between two population means in a study involving independent samples, with normal distribution.

\section{Results}

This study included 27 males (54\%) and 23 females (46\%), the mean gestational age was $37.82 \pm 2.6$ weeks, most cases $(n=40,80 \%)$ were single. and there was no significant difference between CMV and HFOV groups regarding gender, gestational age or singleton Table (1).

Table (1) Socio-demographic data of the studied groups.

\begin{tabular}{lcccccc}
\hline Variable & & CMV Group & HFOV Group & Total & Test & P value \\
& & $\mathbf{N = 2 5}$ & $\mathbf{N = \mathbf { 2 5 }}$ & & & \\
\hline Gender & Male & $14(56 \%)$ & $13(52 \%)$ & $27(54 \%)$ & $\chi^{2}=0.87$ & $\mathrm{P}=0.53$ \\
& Female & $11(44 \%)$ & $12(48 \%)$ & $23(46 \%)$ & & $\mathrm{t}=0.67$ \\
Gestational age & Mean \pm SD & $37.22 \pm 2.6$ & $38.1 \pm 2.7$ & $37.82 \pm 2.6$ & $\mathrm{t}=0.65$ & $\mathrm{P}=0.67$ \\
(Weeks) & Range & $32-41$ & $32-41$ & $32-41$ & & $\mathrm{P}=0.65$ \\
Singleton & Single & $21(84 \%)$ & $19(76 \%)$ & $40(80 \%)$ & $\chi^{2}=0.74$ & \\
& Twins & $2(16 \%)$ & $3(24 \%)$ & $5(20 \%)$ & & \\
\hline
\end{tabular}

t: student t-test; $\chi^{2}$ : Chi-square test; CMV: conventional mechanical ventilation; HFOV: high-frequency oscillatory ventilator.

Regarding Fraction of inspiration $\mathrm{O} 2(\mathrm{FiO} 2)$ of the CMV \& HFOV groups before and after ventilation at different points in time; before ventilation and at 2 hours after ventilation; there was no statistical difference 
between groups regarding the $\mathrm{FiO} 2$. While the $\mathrm{FiO} 2$ was statistically lower in HFOV group than the CMV group at $12 \mathrm{~h}, 24 \mathrm{~h}$, and $48 \mathrm{~h}$ after ventilation, Fig (1).

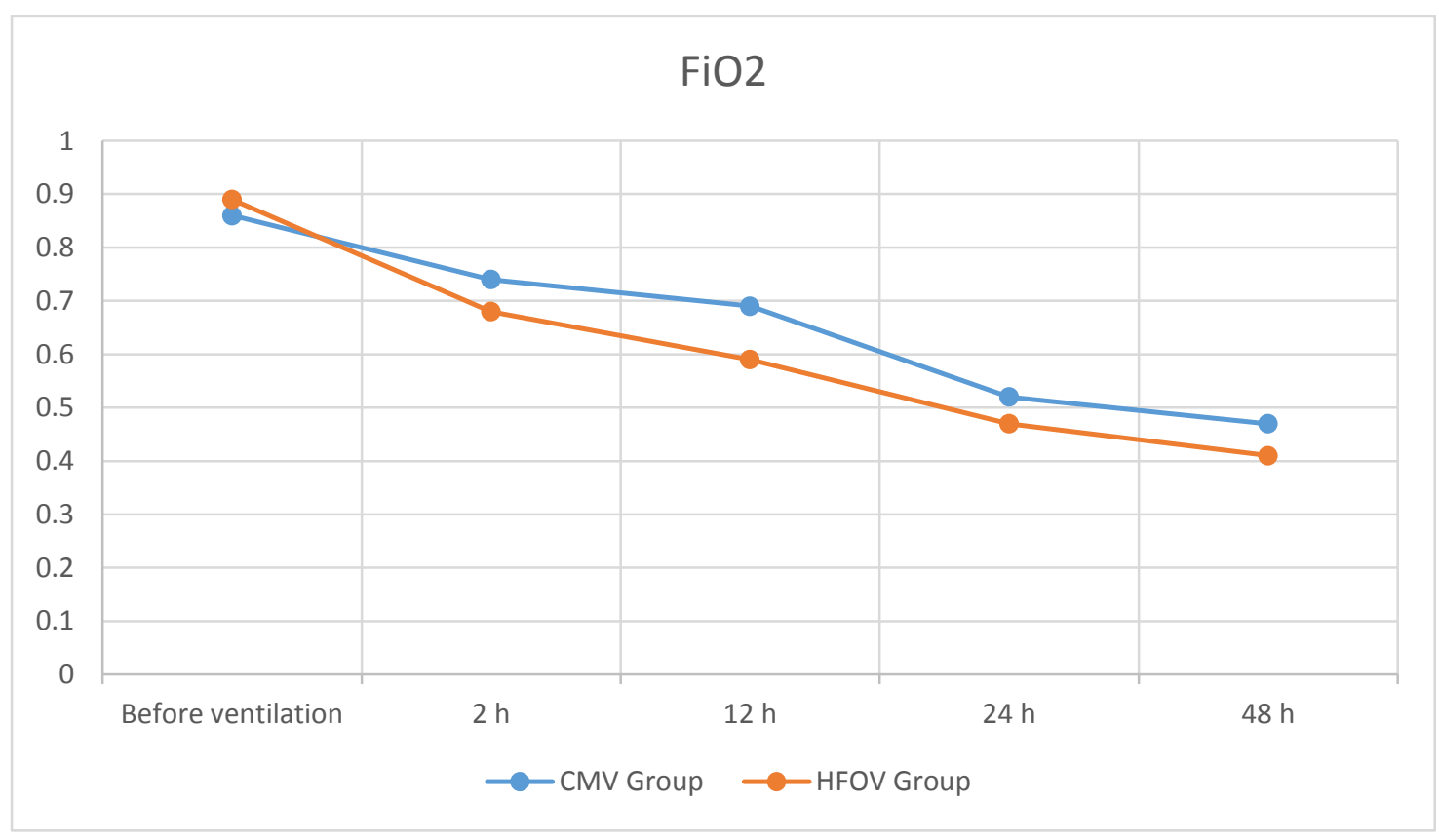

Fig (1) Comparison of the Fraction of inspiration $\mathrm{O} 2(\mathrm{FiO} 2)$ of the CMV \& HFOV groups before and after ventilation at different points in time.

Regarding Oxygenation index (OI) of the CMV \& HFOV groups before and after ventilation at different points in time; before ventilation and at 2 hours after ventilation; there was no statistical difference between groups regarding the OI. While the OI was statistically lower in HFOV group than the CMV group at $12 \mathrm{~h}, 24 \mathrm{~h}$, and $48 \mathrm{~h}$ after ventilation, Fig (2).

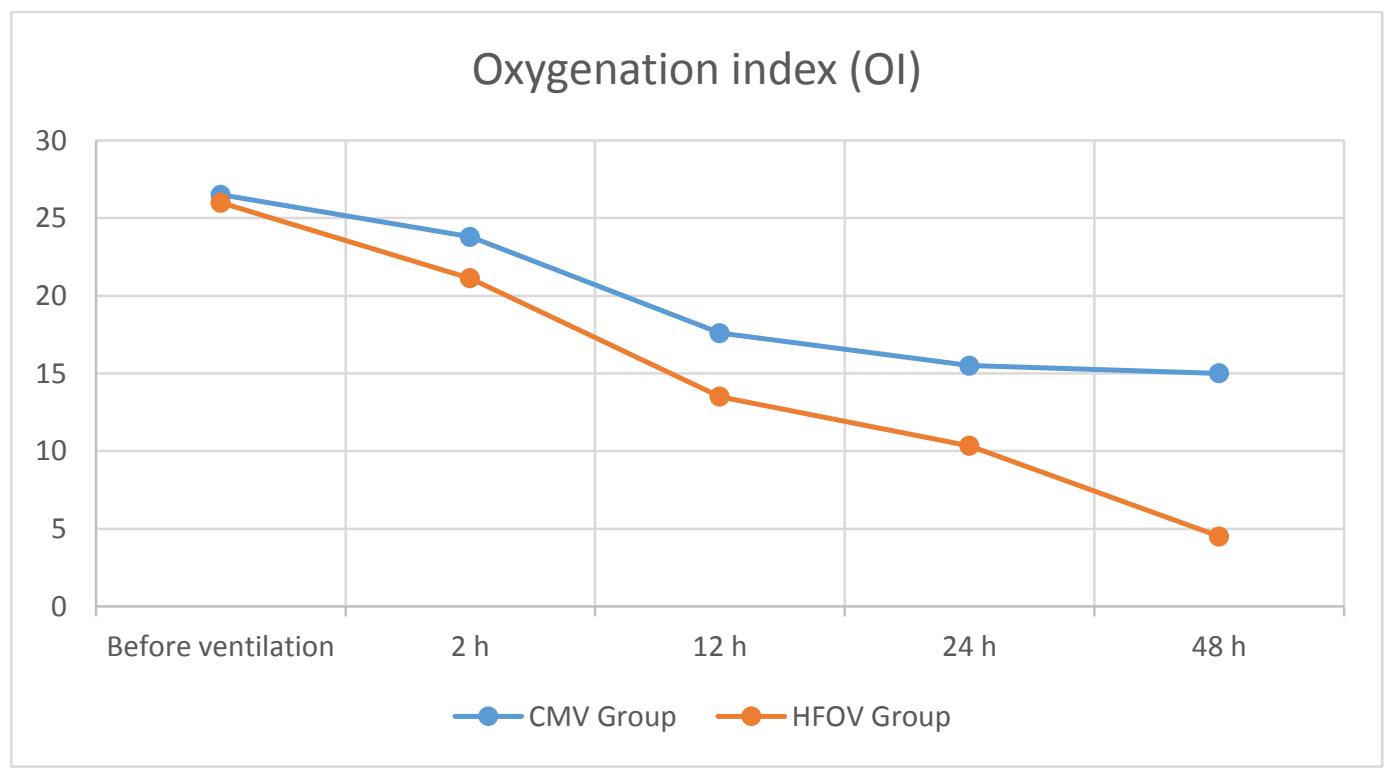

Fig (2) Comparison of the Oxygenation index (OI) of the CMV \& HFOV groups before and after ventilation at different points in time.

Regarding arterial oxygen tension/alveolar arterial oxygen tension $(\mathrm{a} / \mathrm{ApO} 2)$ of the CMV \& HFOV groups before and after ventilation at different points in time; before ventilation, at $2 \mathrm{~h}$, and at $12 \mathrm{~h}$ after ventilation; there was no statistical difference between groups regarding the a/ApO2. While the a/ApO2 was statistically higher in HFOV group than the CMV group at $24 \mathrm{~h}$, and $48 \mathrm{~h}$ after ventilation, Fig (3). 


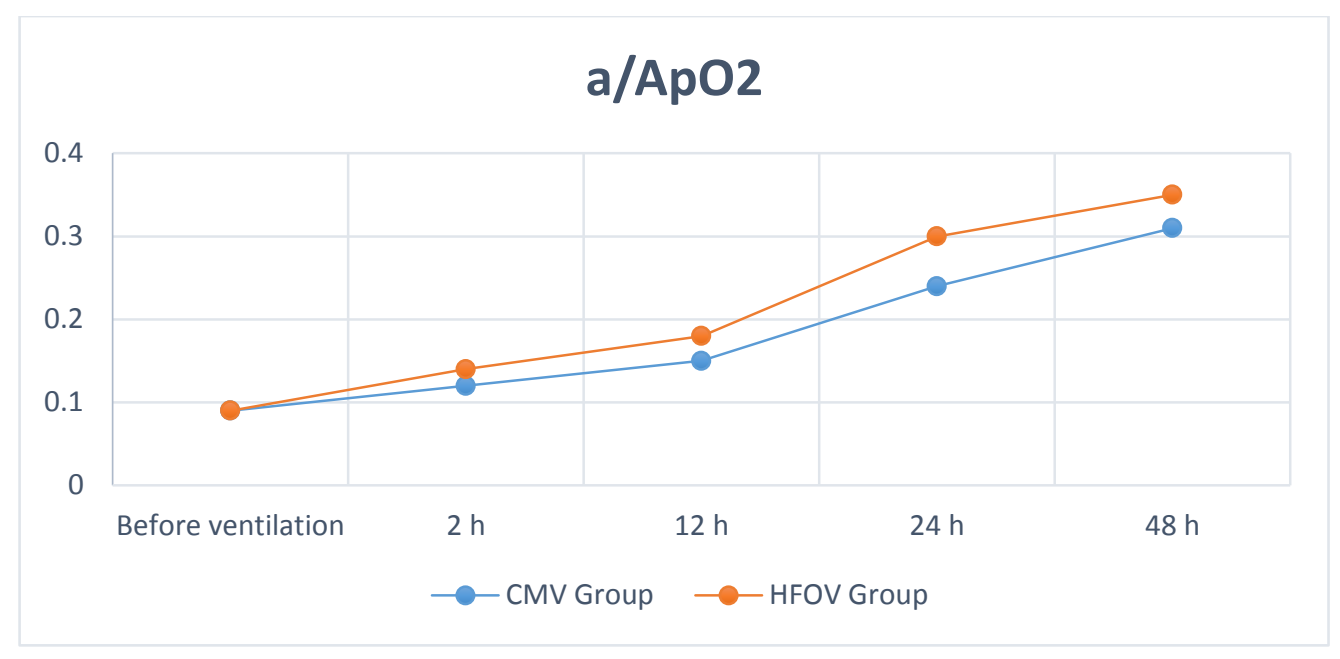

Fig (3) Comparison of the arterial oxygen tension/alveolar arterial oxygen tension (a/ApO2) of the CMV \& HFOV groups before and after ventilation at different points in time

There was no statistical difference between group regarding occurrence of BPD, pneumothorax, sepsis, NEC, IVH or ROP, Table (2).

Table (2) Comparison between groups regarding occurrence of complications.

\begin{tabular}{lccccc}
\hline Variable & & $\begin{array}{c}\text { CMV Group } \\
\mathbf{N = 2 5}\end{array}$ & $\begin{array}{c}\text { HFOV Group } \\
\mathbf{N = 2 5}\end{array}$ & Test & p-value \\
\hline BPD & $\mathbf{N}$ & 1 & 2 & $\chi 2=0.344$ & 0.500 \\
pneumothorax & $\mathbf{\%}$ & $4 \%$ & $8 \%$ & & \\
& $\mathbf{N}$ & 4 & 2 & $\chi 2=0.344$ & 0.500 \\
Sepsis & $\mathbf{\%}$ & $16 \%$ & $8 \%$ & & \\
& $\mathbf{N}$ & 18 & 16 & $\chi 2=0.421$ & 0.313 \\
NEC & $\mathbf{\%}$ & $72 \%$ & $64 \%$ & & - \\
& $\mathbf{N}$ & 1 & 1 & - & \\
IVH & $\mathbf{\%}$ & $2 \%$ & $2 \%$ & & 0.643 \\
& $\mathbf{N}$ & 3 & 2 & $\chi 2=0.499$ & \\
\hline
\end{tabular}

$\chi^{2}$ : Chi-square test; BPD: bronchopulmonary dysplasia; NEC: necrotizing enterocolities; IVH: intraventricular hemorrhage; ROP: retinopathy of prematurity.

CMV had a statistically longer duration of ventilation time, oxygen therapy, and hospitalization than the HFOV group $(\mathrm{p}<0.001$, for all), Fig (4).

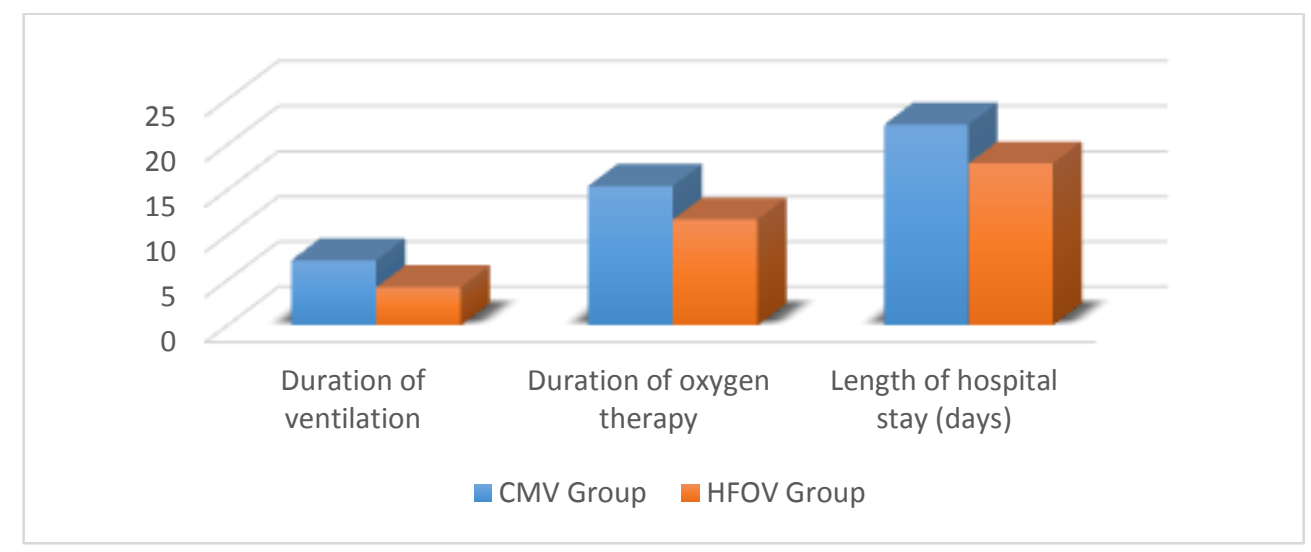

Fig (4) Comparison between CMV \& HFOV groups regarding ventilation time, duration of the oxygen therapy, and duration of hospitalization. 
Surfactant was used in 7 cases $(28 \%)$ of the CMV group and 5 cases $(20 \%)$ of HFOV group, $\mathrm{p}=0.093$. However, mortality rate was higher in CMV group (8 cases, 32\%), than in HOFV group (5 cases, 20\%), there was no significant difference between groups, $\mathrm{p}=0.065$, Table (3).

Table (3) Comparison between groups regarding need of surfactant and mortality.

\begin{tabular}{lccccc}
\hline Variables & & $\begin{array}{c}\text { CMV Group } \\
\mathbf{N = 2 5}\end{array}$ & $\begin{array}{c}\text { HFOV Group } \\
\mathbf{N}=\mathbf{2 5}\end{array}$ & Test & p-value \\
\hline Need of surfactant & $\mathrm{N}$ & 7 & 5 & $\chi^{2}=1.149$ & 0.093 \\
& $\%$ & $28 \%$ & $20 \%$ & & \\
Mortality & $\mathrm{N}$ & 8 & 5 & $\chi 2=1.250$ & 0.065 \\
& $\%$ & $32 \%$ & $20 \%$ & & \\
\hline
\end{tabular}

$\chi^{2}:$ Chi-square test

\section{Discussion}

Regarding Part of motivation $\mathrm{O} 2$ (FiO2) of the CMV and HFOV bunches before ventilation there was no huge contrast between gatherings (the mean $\mathrm{FiO} 2$ in $\mathrm{CMV}$ group $=0.86 \pm 0.21$, in HFOV group $=0.89 \pm 0.12, \mathrm{p}=0.742$ ) and at 2 hours after ventilation; there was no measurable distinction between gatherings (the mean $\mathrm{FiO} 2$ in $\mathrm{CMV}$ group $=0.74 \pm 0.16$, in HFOV group $=0.68 \pm 0.14, p=0.29$ ). While at $12 \mathrm{~h}$ and $24 \mathrm{~h}$ after ventilation the $\mathrm{FiO} 2$ diminished in the two gatherings however was measurably lower in HFOV bunch than the CMV gathering ( $\mathrm{p}=0.045, \mathrm{p}=0.034$, separately). At last at $48 \mathrm{~h}$ after ventilation it was genuinely lower in HFOV bunch than the CMV gathering (the mean $\mathrm{FiO} 2$ in $\mathrm{CMV}$ group $=0.47 \pm 0.21$, in HFOV group $=0.41 \pm 0.11$, $\mathrm{p}=0.02$ ).

As to record (OI) of the CMV and HFOV gatherings; before ventilation there was no huge distinction between gatherings (the mean OI in CMV group $=26.48 \pm 8.27$, in HFOV group $=26.03 \pm 7.81, \mathrm{p}=0.512$ ) and at 2 hours after ventilation; there was no factual contrast between gatherings (the mean $\mathrm{OI}$ in $\mathrm{CMV}$ group $=23.79 \pm 7.27$, in HFOV group=21.13 $\pm 6.29, \mathrm{p}=0.09$ ). While at $12 \mathrm{~h}$ and 24 $h$ after ventilation the OI diminished in the two gatherings yet was genuinely lower in HFOV bunch than the CMV gathering $(\mathrm{p}=0.049, \mathrm{p}=0.013$, separately). At last at $48 \mathrm{~h}$ after ventilation OI was genuinely lower in HFOV bunch than the CMV gathering (the mean OI in CMV group $=15.04 \pm 4.76$, in HFOV group $=4.54 \pm 11.73$, $\mathrm{p}<0.001)$.

As to oxygen pressure/alveolar blood vessel oxygen strain (a/ApO2) of the CMV and HFOV gatherings; before ventilation there was no critical distinction between gatherings (the mean a/ApO2 in CMV group $=0.09 \pm 0.07$, in HFOV group $=0.09 \pm 0.08$, $\mathrm{p}=0.979$ ). What's more, at 2 h. \&4h. after ventilation; a/ApO2 expanded in the two gatherings yet there was no factual distinction between gatherings $(p=0.21 \& \quad p=0.055$, separately). While at $24 \mathrm{~h}$ after ventilation the a/ApO2 was genuinely higher in HFOV bunch than the CMV gathering $(\mathrm{p}=0.022)$. At long last at $48 \mathrm{~h}$ after ventilation a/ApO2 was genuinely higher in HFOV bunch than the $\mathrm{CMV}$ gathering (the mean a/ApO2 in $\mathrm{CMV}$ group $=0.31 \pm 0.07$, in HFOV group $=0.35 \pm 0.06, p=0.003$ ).

Our outcomes were equivalent with [2], who considered the proficiency of high-recurrence oscillatory ventilation joined with pneumonic surfactant in the treatment of neonatal meconium goal disorder. Chen and partners, isolated MAS cases into 3 gatherings as per the diverse treatment approach: bunch 1 regular mechanical ventilation (CMV); bunch $2 \mathrm{HFOV}$; bunch $3 \mathrm{HFOV}+$ pneumonic surfactant. Prior to mechanical ventilation, the three pointers $\mathrm{FiO} 2$, OI and a/ApO2 indicated no huge contrast in all of three gatherings of patients $(\mathrm{P}>$ $0.05)$. Contrasted with bunch 1 , the distinction in all the oxygenation work markers after treatment in gathering 2 and gathering 3 at various focuses as expected, was measurably huge $(\mathrm{P}<0.05)$. also, contrasted with bunch 1 and gathering 2, after the use of PS, there was outstanding lessening in the ventilator markers $\mathrm{FiO} 2$ and MAP in gathering 3 patients at various focuses as expected, and the thing that matters was discovered to be measurably huge $(\mathrm{P}<0.05)$

Concerning complexities in the contemplated gatherings; BPD happened in 1 patient (4\%) of the CMV gathering, and $2(8 \%)$ patients of HFOV gathering. pneumothorax happened in 4 patient $(16 \%)$ of the CMV gathering, and $2(8 \%)$ patients of HFOV gathering. Sepsis happened in 18 patient (72\%) of the CMV gathering, and 16 patients $(64 \%)$ of HFOV gathering. NEC happened in 1 patient (4\%) of the CMV gathering, and 1 patient (4\%) of HFOV gathering. IVH happened in 3 patient $(12 \%)$ of the CMV gathering, and $2(8 \%)$ patients of HFOV gathering. there was no measurable distinction between bunch with respect to event of BPD, pneumothorax, sepsis, NEC, or IVH.

In [14], the most well-known complexity was birth asphyxia in $(37.93 \%, n=22)$ of cases, trailed by Acute Respiratory Failure in $(\mathrm{n}=12,20.68 \%)$ cases. Septicemia was seen in $17.24 \%(\mathrm{n}=10)$ cases, pneumothorax in $6.89 \%(\mathrm{n}=4)$, aspiratory discharge in $3.44 \%(\mathrm{n}=2)$ and ventilated related pneumonia in $3.44 \%$. $(n=2)$ of cases. A few infants had more than one of the previously mentioned intricacies. 28 children $(44.8 \%)$ had confusion other than those referenced previously.

In this examination, CMV had a genuinely longer term of ventilation time (CMV: $7.24 \pm 0.65$ days, HFOV: $4.23 \pm 1.37$ days, $\mathrm{p}<0.001)$, longer oxygen treatment (CMV: 15.39 \pm 2.44 days, HFOV: $11.76 \pm 5.32$ days , $\mathrm{p}<0.001$ ), and longer season of hospitalization (CMV: $22.17 \pm 4.53$ days, HFOV: $17.92 \pm 12.6$ days , $\mathrm{p}<0.001)$. 
Surfactant was utilized in 7 cases $(28 \%)$ of the CMV gathering and 5 cases $(20 \%)$ of HFOV gathering, $\mathrm{p}=0.093$.

In concur with our outcomes, [2] revealed that when contrasted with bunch 1 (CMV), bunch 2 (HFOV) were essentially more limited, the ventilation time (CMV:5 days and HFOV: 3 days), span of the oxygen treatment (CMV :9 days and HFOV: 6 days) and hospitaliza-tion time (CMV :16 days and HFOV: 11 days), the distinctions were discovered to be genuinely huge $(\mathrm{P}<$ 0.05).

Rather than [15], HFOV seems to offer no extra favorable position contrasted with $\mathrm{CV}$ in the therapy of term or close term newborn children with moderate to serious respiratory disappointment, Groups were comparative concerning the frequency of ongoing lung sickness, focal sensory system horribleness, span of mechanical ventilation, and length of hospitalization. There were two early treatment disappointments and two late treatment disappointments in the HFOV gathering and none in the $\mathrm{CV}$ gathering.

Anyway in [11], The middle terms of ventilation and oxygen supplementation were was 22 hours and 66 hours, separately. Four out of $170(2.3 \%)$ patients got surfactant treatment for MAS. The middle term of emergency clinic stay among the individuals who endure was 168 hours and the middle chance to mortality was 24 hours.

In [16] learn about the connection between incendiary records and the result of meconium yearning disorder, the middle scope of affirmation at NICU was 8 days, middle scope of mechanical ventilator was 4 days and middle span of oxygen treatment was 3 days.

In this examination, in any case, death rate was higher in CMV gathering ( 8 cases, $32 \%$ ), than in HOFV gathering ( 5 cases, 20\%), there was no huge distinction between gatherings, $\mathrm{p}=0.065$.

In the examination by [11], they contemplated the indicators of mortality in youngsters with meconium yearning condition, Out of 172 included children, 44 (26\%) kicked the bucket. In [14] study, Mortality happened in 06 cases $(10.34 \%$ of the considered gathering with MAS). In any case, in Gangu Dhillis [14] study, just 18 cases $(31.03 \%)$ required ventilator uphold where sign was birth asphyxia, intense respiratory disappointment or different difficulties like pneumothorax. Out of 18 ventilated children, 06 infants kicked the bucket and 12 children endure and was released.

While in [2], the death rate was $4.5 \%$ in CMV gathering (1 patient) and $5.5 \%$ in HFOV gathering (1 patient), there was no huge measurable contrast in the death rate between gatherings.

This investigation was restricted by the little example size, which didn't take into consideration a superior examination, anyway our outcomes demonstrated that HFOV seems to offer extra favorable circumstances contrasted with CMV in the treatment of Meconium desire disorder, by diminishing the term of mechanical ventilation, and length of hospitalization, yet it didn't influence the mortality rate.

\section{Conclusion}

Meconium desire disorder (MAS) is mind boggling respiratory infection of the term and close term youngster, which keeps on putting a significant weight on neonatal inten $\neg$ sive consideration assets around the world. Our outcomes indicated that HFOV seems to offer extra focal points contrasted with CMV in the treatment of MAS, by lessening the term of mechanical ventilation, and span of hospitalization, anyway it didn't influence the death rate.

\section{References}

[1] K. Swarnam, A. S. Soraisham, S. Sivanandan, "Advances in the management of meconium aspiration syndrome," Int. J. Pediatr, Vol. 2012(3), PP. 65-64, 2011.

[2] D. M. Chen, L. Q. Wu, R.-Q. Wang, "Efficiency of high-frequency oscillatory ventilation combined with pulmonary surfactant in the treatment of neonatal meconium aspiration syndrome," Int. J. Clin. Exp. Med, Vol. 8(8), PP. 144-90, 2015.

[3] E. K. Hutton , J. Thorpe, "Consequences of meconium stained amniotic fluid: what does the evidence tell us?," Early Hum. Dev, Vol. 90(7), PP. 333-339, 2014.

[4] R. Y. Bhat , A. Rao, "Meconium-stained amniotic fluid and meconium aspiration syndrome: a prospective study," Ann. Trop. Paediatr, Vol. 28(3), PP. 199-203, 2008.

[5] P. C. Rimensberger, S. M. Schulzke, D. Tingay, B. S. Von Ungern-Sternberg, "Pediatric and neonatal mechanical ventilation," From Basics to Clin, Vol. 5(4), PP. 56-71, 2015.

[6] P. A. Dargaville, "Respiratory support in meconium aspiration syndrome: a practical guide," Int. J. Pediatr, Vol. 2012(6), PP. 87-90, 2012.

[7] M. Moniz , "High-frequency oscillatory ventilation in children: a 10-year experience," J. Pediatr. (Rio. J), Vol. 89(1), PP. 48-55, 2013.

[8] O. Erdeve ,"An observational, prospective, multicenter study on rescue high-frequency oscillatory ventilation in neonates failing with conventional ventilation," PLoS One, Vol. 14(6), PP. 90-102, 2019.

[9] A. Pellicano, D. G. Tingay, J. F. Mills, S. Fasulakis, C. J. Morley, P. A. Dargaville, "Comparison of four methods of lung volume recruitment during high frequency oscillatory ventilation," Intensive Care Med, Vol. 35(11), PP. 19-90, 2009.

[10]R. Gupta , D. Rosen, "Paediatric mechanical ventilation in the intensive care unit," Bja Educ, Vol. 16(12), PP. 422-426, 2016.

[11]D. Louis, V. Sundaram, K. Mukhopadhyay, S. Dutta, P. Kumar, "Predictors of mortality in neonates with meconium aspiration syndrome," Indian Pediatr, Vol. 51(8), PP. 637-640, 2014. 
[12] M. Rawat , "Oxygen saturation index and severity of hypoxic respiratory failure," Neonatology, Vol. 107(3), PP. 161-166, 2015.

[13] J. Te Hsu , "Prognostic value of arterial/alveolar oxygen tension ratio (a/APO2) in acute pulmonary embolism," Circ. J, Vol. 71(10), PP. 1560-1566, 2007.

[14] R. N. Gangu Dhilli , P. A., "Study of clinical profile of meconium aspiration syndrome in relation to gestational age and birth weight and their immediate outcome at Narayana Medical College Hospital, Nellore, India," Int. J. Contemp. Pediatr; Vol 4(6),PP. 18203-2349,2017.
[15]M. A. Rojas ,"Randomized, multicenter trial of conventional ventilation versus high-frequency oscillatory ventilation for the early management of respiratory failure in term or near-term infants in Colombia," J. Perinatol, Vol. 25(11), PP. 720-724, 2005.

[16] M. A. MOHAMED , M. A. MOHAMED, "Relation Between Inflammatory Indices and The Outcome of Meconium Aspiration Syndrome," Relation, Vol. 22 (2), PP. 32-43, 2018. 